\title{
Energy-Efficient Resource Allocation in Cooperative OFDMA Systems
}

\author{
Fatma Irem Sökmen \\ Department of Electrical and \\ Electronics Engineering \\ TOBB University of Economics and Technology \\ Ankara, Turkey \\ Email: fsokmen@etu.edu.tr
}

\author{
Tolga Girici \\ Department of Electrical and \\ Electronics Engineering \\ TOBB University of Economics and Technology \\ Ankara, Turkey \\ Email: tgirici@etu.edu.tr
}

\begin{abstract}
In this work we study framed transmission in an OFDMA based relayed wireless access system that utilize cooperative diversity. We consider a system of a source, a relay and a number of destination nodes, where the nodes can combine the signals coming from the source and relay. We are interested in the case that the source and relay only have the information of path loss and slow fading. The goal in resource allocation is to allocate resources (time slots, subchannels and power) in an energy efficient manner given target rates and target outage probability. Numerical results show that cooperative relaying requires significantly less energy than noncooperative relaying. Adjusting the source and relay transmission durations has a marginal effect on the performance, although it results in a significant increase in complexity.
\end{abstract}

\section{INTRODUCTION}

In this work we consider the transmission of a source node to a number of destination nodes, with the help of a relay node. Transmitters use a multichannel transmission scheme such as Orthogonal Frequency Division Multiplexing (OFDM). OFDM is the underlying transmission technology in current wireless technologies such as Wi-Fi, WiMax and LTE. OFDM provides immunity to multipath fading and intersymbol interference, hence higher signal to interference plus noise ratio (SINR). Besides, it can be used as a multiple access scheme (OFDMA) in order to exploit multichannel and multiuser diversity by judiciously allocating subchannels to users.

Relays are deployed in order to improve coverage, throughput and energy-efficiency in broadband wireless access [1] and multihop wireless networks [2]. These relays can also be used to mimic multiple antenna systems and take advantage of cooperative diversity. For example in the downlink, a destination node can combine signals coming from the source and relay in order to improve the SINR. There are two practical and well-known forms of cooperative diversity, which are Amplify and Forward (AF) and Decode and Forward (DF) [3]. Using OFDM and relays along with cooperative transmission can provide more degrees of freedom by exploiting adaptive subchannel, power and time allocation. Besides relays in wireless networks are usually half-duplex, which necessitates a TDMA-type of time sharing mechanism between the source and relay. Time allocation adds another dimension to the resource allocation problem. In a cooperative relayed cellular system, time is divided into transmission frames. In the first part of the frame the source broadcasts the information, which is both heard from the relay and the destination. After the relay receives the signal it transmits it to the destination where the signals are combined at the MS. In order to achieve this the source-relay and relay-destination transmission durations and signaling must be equal [3]. On the other hand, some destination nodes may be so close to the source that direct transmission by the source using the whole frame duration may be more efficient.

OFDMA-based cooperative resource allocation can be made for various purposes such as maximizing the throughput or proportional fairness subject to power constraints or reducing power expenditure subject to some rate requirements. In the previous literature on the subject mostly throughput maximization was studied. In [4] and [5] the goal is to maximize the throughput. Subchannel allocation at the source-relay and relay-destination channels should be jointly considered in order to maximize capacity. In these works power allocation was also considered. In [6] a three terminal system is considered and adaptive power loading is studied in order to minimize bit error rate. Joint resource allocation and selection of cooperation strategies and relay to be used in multi relay systems has been studied in [7]. In [8], the authors considered proportional fair resource allocation in an OFDM based cooperative relayed cellular system. They studied different subframe allocation and cooperative diversity schemes. In this work it is shown that time durations (subframes) allocated for the base and relay stations is a very important factor in performance improvement.

Most of the previous works on OFDM based resource allocation assume that the channel conditions are perfectly known at each scheduling interval (that is, a frame or a slot). In reality, obtaining the channel state information for each user-subchannel pair is not very practical. Especially in the presence of relays, channel information of users should be forwarded to the source (base station), creating further communication overhead. A more practical assumption is that the users measure the channel state for some time and feedback the channel information averaged over that certain duration. 
Channel state is usually a combination of path loss, lognormal shadowing and fast fading (which is usually Rayleigh distributed and frequency selective). Time-averaged channel gain captures the slow components of the fading, which are the path loss and shadowing. In this case scheduling decisions have to be made under channel uncertainty. Then, given a modulation and coding rate there is a nonzero probability of outage [3] where the received SNR falls below a required level. In [19] a single source single destination and multiplerelay system was considered. There is a fixed target rate and each transmitted subchannel is relayed by the best relay such that the achievable rate is maximized. The probability that achievable rate falls below the target (i.e. outage) is analyzed. However, it is still assumed that exact channel conditions are known. On the other hand in [11] the authors consider multiple users transmitting to a center with the help of a relay. It is assumed that channel condition is not known and the outage probability of the proposed transmission protocol is probabilistically analyzed. The optimum relay location is computed based on the analytical result.

In this work, we consider the problem of resource allocation for total energy expenditure minimization in a cooperative system of a source, a relay multiple users. The resource are subchannels, power, and transmission durations of source and relay at each subchannel (i.e. subframe durations). We assume that the resource allocation is made by the source and it knows only the average channel gains. Minimum-power resource allocation in OFDM based systems was previously studied for systems without relays [14], [13] and with non-cooperative relaying in [16] and [15]. In cooperative relayed OFDMA systems power minimization was first considered in [18]. Here the authors consider a two-user, one-base station uplink scenario. The users help each other using an AF scheme. Power minimization in a single source-destination pair and multi-relay system is studied in [19]. The authors propose a scheme that reallocates subchannels at the relay in order to improve the performance. Differently from this work we assume that fast fading is not known and outage probability is a performance target. In our work the aim in resource allocation is to minimize the total transmission energy in a frame duration while satisfying rate and outage probability constraints. We are interested in the performance improvement by 1) Cooperative transmission of source and relay in the absence of perfect channel information and outages 2) Optimally determining the subframe duration for the source-relay and relay-destination transmissions. The rest of the paper is organized as follows; in section II the system model is introduced. In Section III the various transmission methods have been described and analyzed. Section IV includes the numerical evaluation results and Section $\mathrm{V}$ gives the conclusions we have reached in this paper.

\section{System Model}

We consider a source node (e.g. a base station), a relay and multiple destination nodes. The relay uses Decode and Forward (DF) or Amplify and Forward (AF) in relaying the information from the source to destinations. The total bandwidth of $W \mathrm{~Hz}$ is divided into $K$ subchannels each having the bandwidth $W_{s}$. These channel states are assumed to be constant during a transmission frame. Each user experiences path loss , log-normal shadowing and frequency selective and fast Rayleigh fading. In this system there are three types of transmission channels which are the (S)ource-(R)elay, (R)elay(D)estination and (S)ource-(D)estination channels. The destinations can also overhear the transmissions from the S-R channel. Let $g_{n}^{S D}, g^{S R}, g_{n}^{R D}$ be the combination of pathloss and slow (shadow) fading for destination $n$. The source node perfectly knows these average values for all nodes and the relay and performs the resource allocation based on these values. Let $h_{n, k}^{S D}, h_{k}^{S R}, h_{n, k}^{R D}$ be the frequency selective Rayleigh fading for user $n$ and subchannel $k$. Note that $g^{S R}$ and $h_{k}^{S R}$ are the same for all users, because there is a single relay. The users are assumed to have constant channel gains during a transmission frame, which is of duration $T_{f}$ and divided into time slots of duration $T_{s}$. Let $R_{n}$ be the rate requirement and $\kappa_{n}$ be the number of subchannels allocated to user $n$. Given $\kappa_{n}$ we assume that there exists a modulation-coding pair and the transmitters use that pair. We use the information theoretic achievable rate expressions [3] and outage happens if the achievable rate is lower than the target rate. We also assume that information transmitted using a subchannel by the source is transmitted using the same subchannel by the relay. Outage probability can be reduced by increasing the energy expenditure. The goal is to satisfy the user rate and outage probability requirements by minimum energy.

\section{Resource Allocation Schemes}

As the frequency selective fast fading is not known and path loss and shadowing are assumed to be subchannelindependent, all subchannels are equivalent and therefore power is equally distributed to the allocated subchannels. Target rate is equally shared among the subchannels and outage happens if any of the subchannels experience outage. This implies that each subchannel of a user has equal probability of outage. Given the target outage probability $P_{\text {out }}$, the target outage probability of user $n$ for each subchannel becomes $P_{\text {out }}^{s, n}=1-\left(1-P_{\text {out }}\right)^{\frac{1}{\kappa_{n}}}$. For low outage probabilities, this can be approximated by $\frac{P_{\text {out }}}{\kappa_{n}}$. In fact, this is always slightly less than the actual value, therefore it is more conservative. We consider four different transmission (and frame allocation) policies, as illustrated in Figure 1. As illustrated in the figure, for all policies, a number of subchannels are allocated to each user. For relayed users, allocated subchannels are divided into two subframes, where the source and relay transmit. There are also direct users, which don't use the relay. The details are described below;

A. Non-Cooperative Relaying with Time Optimization (NCRTO)

Figure 1(a) shows the frame model for the case of five users. For destination $n, \kappa_{n}$ subchannels are allocated and the frame time $T_{f}$ is divided into two for the source and relay 
(a)

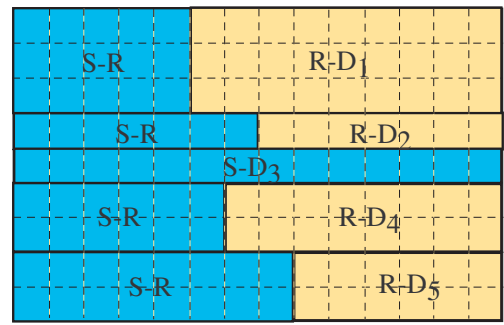

$\alpha_{1}=5 / 14, \kappa_{1}=3$

$\alpha_{2}=7 / 14, \kappa_{n}=1$

$\alpha_{3}=1, \kappa_{n}=1$

$\alpha_{4}=6 / 14, \kappa_{n}=2$

$\alpha_{5}=8 / 14, \kappa_{n}=2$

(b)

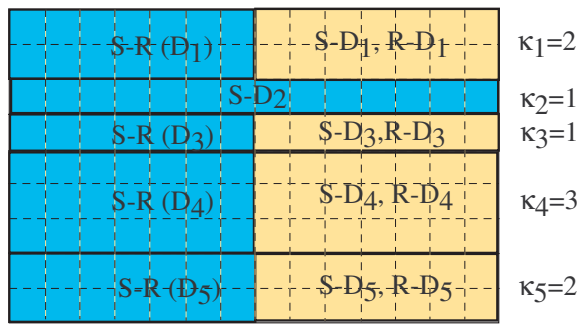

(c)

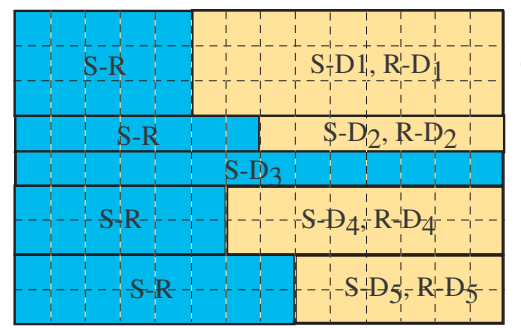

$\alpha_{1}=5 / 14, \kappa_{1}=3$

$\alpha_{2}=7 / 14, \kappa_{n}=1$

$\alpha_{3}=1, \kappa_{n}=1$

$\alpha_{4}=6 / 14, \kappa_{n}=2$

$\alpha_{5}=8 / 14, \kappa_{n}=2$

Fig. 1. Transmission policies and corresponding frame models. (a)NonCooperative Relaying with Time Optimization (NCR-TO) (b) Cooperative Relaying (Using Decode and Forward (DF) or Amplify and Forward (AF) ) without Time Optimization (c)Decode and Forward Relaying with Time Optimization (DF-TO)

transmissions. In the first subframe (of duration $\alpha_{n} T_{f}$ ) the source broadcasts the information, but is only decoded by the relay. Once the relay gathers the information it decodes, it re-encodes and forwards it to the destination in the second subframe (of duration $\left(1-\alpha_{n}\right) T_{f}$ ). Here $\alpha_{n}$ can be optimized ( $\alpha_{n} T_{f}$ has to be an integer multiple of time slot duration) for all $n$ to further improve the resource utilization. On the other hand, if it is more energy-efficient, some users can be set as direct users and served directly by the source using all the frame duration (e.g. node $D_{3}$ ). Let $e_{n}^{S R}$ and $e_{n}^{R D}$ be the energy expenditures by the source and relay for destination $n$. Then $\frac{e_{n}^{S R}}{\alpha_{n} T_{f}}$ and $\frac{e_{n}^{R D}}{\left(1-\alpha_{n}\right) T_{f}}$ become the power expenditure by the source and relay for user $n$. The aim is to find for all users the energy expenditures that satisfy the target outage probability and rate requirements with minimal total energy. As mentioned above, $\frac{P_{\text {out }}}{\kappa_{n}}$ is the target outage probability for any subchannel allocated to destination $n$. Without using subscripts, let $h^{S R}$ and $h^{R D}$ be the Rayleigh fading gain for any subchannel allocated to user $n$. We assume that $h^{S D}, h^{R D}$ and $h^{R D}$ are independent and exponentially distributed with unit mean. For each allocated subchannel, the outage probability equation is,

$$
\begin{gathered}
\frac{P_{\text {out }}}{\kappa_{n}}=\operatorname{Pr}\left\{\operatorname { m i n } \left\{\alpha_{n} \log _{2}\left(1+\frac{e_{n}^{S R} g^{S R} h^{S R}}{\kappa_{n} N_{0} W_{s} \alpha_{n} T_{f}}\right)\right.\right. \\
\left.\left.,\left(1-\alpha_{n}\right) \log _{2}\left(1+\frac{e_{n}^{R D} g_{n}^{R D} h^{R D}}{\kappa_{n} N_{0} W_{s}\left(1-\alpha_{n}\right) T_{f}}\right)\right\}<\frac{R_{n}}{\kappa_{n} W_{s} T_{f}}\right\}
\end{gathered}
$$

Here $\frac{e_{n}^{S R}}{\kappa_{n} \alpha_{n} T_{f}}$ and $\frac{e_{n}^{S R}}{\kappa_{n}\left(1-\alpha_{n}\right) T_{f}}$ are the power expenditures for the S-R and R-D links in one of the allocated subchannels. For simplicity, let's define,

$$
\begin{aligned}
& e_{n}^{01}\left(\kappa_{n}, \alpha_{n}\right)=\alpha_{n} T_{f} \kappa_{n} N_{0} W_{s}\left(2^{\frac{R_{n}}{\kappa_{n} W_{s} T_{f} \alpha_{n}}}-1\right) \\
& e_{n}^{02}\left(\kappa_{n}, \alpha_{n}\right)=\left(1-\alpha_{n}\right) T_{f} \kappa_{n} N_{0} W_{s}\left(2^{\frac{R_{n}}{\kappa_{n} W_{s} T_{f}\left(1-\alpha_{n}\right)}}-1\right)
\end{aligned}
$$

After some rearrangements on the outage equation, we obtain,

$$
-\ln \left(1-\frac{P_{\text {out }}}{\kappa_{n}}\right)=\frac{e_{n}^{01}\left(\kappa_{n}, \alpha_{n}\right)}{e_{n}^{S R} g^{S R}}+\frac{e_{n}^{02}\left(\kappa_{n}, \alpha_{n}\right)}{e_{n}^{R D} g_{n}^{R D}}
$$

We need to find the energy expenditures $e_{n}^{S R}$ and $e_{n}^{R D}$ that satisfy the above equation with a minimal total, for energy efficiency. Using convex optimization techniques, the optimal energy expenditures are,

$$
\begin{aligned}
& e_{n}^{S R}\left(\kappa_{n}, \alpha_{n}\right)= \frac{\frac{e_{n}^{01}\left(\kappa_{n}, \alpha_{n}\right)}{g^{S R}}+\sqrt{\frac{e_{n}^{01}\left(\kappa_{n}, \alpha_{n}\right) e_{n}^{02}\left(\kappa_{n}, \alpha_{n}\right)}{g^{S R} g_{n}^{R D}}}}{-\ln \left(1-\frac{P_{\text {out }}}{\kappa_{n}}\right)} \\
& e_{n}^{R D}\left(\kappa_{n}, \alpha_{n}\right)=\frac{\frac{e_{n}^{02}\left(\kappa_{n}, \alpha_{n}\right)}{g_{n}^{R D}}+\sqrt{\frac{e_{n}^{01}\left(\kappa_{n}, \alpha_{n}\right) e_{n}^{02}\left(\kappa_{n}, \alpha_{n}\right)}{g^{S R} g_{n}^{R D}}}}{-\ln \left(1-\frac{P_{\text {out }}}{\kappa_{n}}\right)}
\end{aligned}
$$

The total energy expenditure using the relay is,

$$
\begin{aligned}
e_{n}^{\text {relay }}\left(\kappa_{n}, \alpha_{n}\right)= & e_{n}^{S R}\left(\kappa_{n}, \alpha_{n}\right)+e_{n}^{R D}\left(\kappa_{n}, \alpha_{n}\right) \\
& =\frac{\left(\sqrt{\frac{e_{n}^{01}\left(\kappa_{n}, \alpha_{n}\right)}{g^{S R}}}+\sqrt{\frac{e_{n}^{02}\left(\kappa_{n}, \alpha_{n}\right)}{g_{n}^{R D}}}\right)^{2}}{-\ln \left(1-\frac{P_{\text {out }}}{\kappa_{n}}\right)}
\end{aligned}
$$

Time-optimized energy expenditure can be found by optimizing $\alpha_{n}$. Let $e_{n}^{\text {relay }}\left(\kappa_{n}, \alpha_{n}^{*}\right)=e_{n}^{S R}\left(\kappa_{n}, \alpha_{n}^{*}\right)+e_{n}^{R D}\left(\kappa_{n}, \alpha_{n}^{*}\right)$ be the time optimized total energy expenditure for a relayed user.

In case of direct transmission that uses the whole frame duration, the total energy expenditure is $e_{n}^{S D}$, which becomes

$$
e_{n}^{\text {direct }}\left(\kappa_{n}\right)=e_{n}^{S D}\left(\kappa_{n}\right)=\frac{T_{f} \kappa_{n} N_{o} W_{s}\left(2^{\frac{R_{n}}{W_{s} T_{f} \kappa_{n}}}-1\right)}{-\ln \left(1-\frac{P_{\text {out }}}{\kappa_{n}}\right) g_{n}^{S D}}
$$

Since the frame is not divided in case of direct transmission, the energy is not a function of $\alpha_{n}$. If $e_{n}^{\text {direct }}\left(\kappa_{n}\right)$ is smaller than $e_{n}^{\text {relay }}\left(\kappa_{n}, \alpha_{n}^{*}\right)$, then $e_{n}^{S R}\left(\kappa_{n}\right)=e_{n}^{R D}\left(\kappa_{n}\right)=0$ and $e_{n}^{\text {direct }}\left(\kappa_{n}\right)$ is as in the above equation. 


\section{B. Decode and Forward based Relaying (DF)}

In this scheme the destination nodes can combine the signals coming from the source and relay. As seen in Figure 1(b), if relayed transmission is used, then the frame is divided into two equal subframes. In the first subframe the source transmits to the relay using energy $e_{n}^{S R}$ and the destination overhears it. In the second subframe both source and relay transmit simultaneously with energy $e_{n}^{S D}$ and $e_{n}^{R D}$, using space time coding [8],[9]. If it is more energy-efficient, direct transmission can also be used as is the case for destination $D_{2}$. These energy values are divided into $\kappa_{n}$ to get the energy used in one subchannel. Now let us define for simplicity $e_{n}^{0}\left(\kappa_{n}\right)=0.5 T_{f} \kappa_{n} N_{o} W_{s}\left(2^{\frac{2 R_{n}}{W_{s} T_{f} \kappa_{n}}}-1\right)$. The outage probability equation becomes as follows [3],

$$
\begin{aligned}
& \frac{P_{\text {out }}}{\kappa_{n}}=\operatorname{Pr}\left\{\operatorname { m i n } \left(e_{n}^{S R} g^{S R} h^{S R}\right.\right. \\
& \left.\left.\quad,\left(e_{n}^{S R}+e_{n}^{S D}\right) g_{n}^{S D} h^{S D}+e_{n}^{R D} g_{n}^{R D} h^{R D}\right)<e_{n}^{0}\left(\kappa_{n}\right)\right\}
\end{aligned}
$$

This can be rewritten as,

$$
\begin{aligned}
& \frac{P_{\text {out }}}{\kappa_{n}}=\operatorname{Pr}\left\{e_{n}^{S R} g^{S R} h^{S R}<e_{n}^{0}\left(\kappa_{n}\right)\right\} \\
& \quad+\operatorname{Pr}\left\{e_{n}^{S R} g^{S R} h^{S R} \geq e_{n}^{0}\left(\kappa_{n}\right)\right\} \\
& \times \operatorname{Pr}\left\{\left(e_{n}^{S R}+e_{n}^{S D}\right) g_{n}^{S D} h^{S D}+e_{n}^{R D} g_{n}^{R D} h^{R D}<e_{n}^{0}\left(\kappa_{n}\right)\right\}
\end{aligned}
$$

We need to find $e_{n}^{S R}, e_{n}^{S D}$ and $e_{n}^{R D}$ that satisfies the above equation with minimum total. In order to find the energy values more simply (sacrificing optimality), we give the two added terms in the above equation equal probability $\frac{P_{\text {out }}}{2 \kappa_{n}}$. Targeting $\operatorname{Pr}\left\{e_{n}^{S R} g^{S R} h^{S R}<e_{n}^{0}\left(\kappa_{n}\right)\right\}=\frac{P_{\text {out }}}{2 \kappa_{n}}$ we directly find $e_{n}^{S R}$ as

$$
e_{n}^{S R}\left(\kappa_{n}\right)=\frac{e_{n}^{0}\left(\kappa_{n}\right)}{-\ln \left(1-\frac{P_{\text {out }}}{2 \kappa_{n}}\right) g^{S R}}
$$

Equation (8) implies $\operatorname{Pr}\left\{e_{n}^{S R} g^{S R} h^{S R}>e_{n}^{0}\left(\kappa_{n}\right)\right\}=1-$ $\frac{P_{\text {out }}}{2 \kappa_{n}}$. Using this we find

$$
\begin{aligned}
& \frac{1}{\frac{2 \kappa_{n}}{P_{\text {out }}}-1} \\
= & \operatorname{Pr}\left\{\left(e_{n}^{S R}+e_{n}^{S D}\right) g_{n}^{S D} h^{S D}+e_{n}^{R D} g_{n}^{R D} h^{R D}<e_{n}^{0}\left(\kappa_{n}\right)\right\}
\end{aligned}
$$

This involves the summation of two exponentially distributed random variables being smaller than a constant. We can write this relation as,

$$
\frac{1}{\frac{2 \kappa_{n}}{P_{\text {out }}}-1}=\frac{\frac{1-e^{-\frac{e_{n}^{0}\left(\kappa_{n}\right)}{\left(e_{n}^{S R}+e_{n}^{S D}\right) g_{n}^{S D}}}}{e_{n}^{R D} g_{n}^{R D}}-\frac{1-e^{-\frac{e_{n}^{0}\left(\kappa_{n}\right)}{e_{n}^{R D} g_{n}^{R D}}}}{\left(e_{n}^{S R}+e_{n}^{S D}\right) g_{n}^{S D}}}{\frac{1}{e_{n}^{R D} g_{n}^{R D}}-\frac{1}{\left(e_{n}^{S R}+e_{n}^{S D}\right) g_{n}^{S D}}}
$$

For a low target outage probability and high SNR, we can assume that $e^{-\frac{\kappa_{n} e_{n}^{0}\left(\kappa_{n}\right)}{\left(e_{n}^{S R}+e_{n}^{S D}\right) g_{n}^{S D}}}$ and $e^{-\frac{\kappa_{n} e_{n}^{0}\left(\kappa_{n}\right)}{e_{n}^{R D} g_{n}^{R D}}}$ are close to zero. At this point we make the approximation $e^{-x} \simeq 1-x+x^{2}$ and write the following

$$
\frac{1}{\frac{2 \kappa_{n}}{P_{\text {out }}}-1}=\frac{\left(e_{n}^{0}\left(\kappa_{n}\right)\right)^{2}}{2\left(e_{n}^{S R}+e_{n}^{S D}\right) g_{n}^{S D} e_{n}^{R D} g_{n}^{R D}}
$$

If we use the approximation in (10) then

$$
\left(e_{n}^{S R}+e_{n}^{S D}\right) e_{n}^{R D}=\frac{\left(e_{n}^{0}\left(\kappa_{n}\right)\right)^{2}\left(\frac{2 \kappa_{n}}{P_{\text {out }}}-1\right)}{2 g_{n}^{S D} g_{n}^{R D}}
$$

Here we have the requirement that the product of two values $e_{n}^{S R}+e_{n}^{S D}$ and $e_{n}^{R D}$ are equal to a constant. We need to find the two values such that $e_{n}^{S R}+e_{n}^{S D}+e_{n}^{R D}$ is minimal. Normally this implies that $e_{n}^{S R}+e_{n}^{S D}=e_{n}^{R D}$, however the actual values of $e_{n}^{S R}, e_{n}^{S D}$ and $e_{n}^{R D}$ also depend on (8). These values are found as follows,

- $e_{n}^{S R}\left(\kappa_{n}\right)$ is found according to (8)

$$
\begin{aligned}
& \text { - If } \frac{e_{n}^{0}\left(\kappa_{n}\right)}{-\ln \left(1-\frac{P_{\text {out }}}{2 \kappa_{n}}\right) g^{S R}}>e_{n}^{0}\left(\kappa_{n}\right) \sqrt{\frac{\frac{2 \kappa_{n}}{P_{\text {out }}}-1}{2 g_{n}^{S D} g_{n}^{R D}}} \text { then } \\
& e_{n}^{S D}\left(\kappa_{n}\right)=0 \\
& e_{n}^{R D}\left(\kappa_{n}\right)=\frac{-\ln \left(1-\frac{P_{\text {out }}}{2 \kappa_{n}}\right)\left(\frac{2 \kappa_{n}}{P_{\text {out }}}-1\right) e_{n}^{0}\left(\kappa_{n}\right) g^{S R}(13)}{2 g_{n}^{S D} g_{n}^{R D}} \\
& \text { - If } \frac{e_{n}^{0}\left(\kappa_{n}\right)}{-\ln \left(1-\frac{P_{\text {out }}}{2 \kappa_{n}}\right) g^{S R}}<e_{n}^{0}\left(\kappa_{n}\right) \sqrt{\frac{\frac{2 \kappa_{n}}{P_{\text {out }}}-1}{2 g_{n}^{S D} g_{n}^{R D}}} \text { then } \\
& e_{n}^{S D}\left(\kappa_{n}\right)=e_{n}^{0}\left(\kappa_{n}\right) \sqrt{\frac{\frac{2 \kappa_{n}}{P_{\text {out }}}-1}{2 g_{n}^{S D} g_{n}^{R D}}}-\frac{e_{n}^{0}\left(\kappa_{n}\right)}{-\ln \left(1-\frac{P_{\text {out }}}{2 \kappa_{n}}\right) g^{S R}} \\
& e_{n}^{R D}\left(\kappa_{n}\right)=e_{n}^{0}\left(\kappa_{n}\right) \sqrt{\frac{\frac{2 \kappa_{n}}{P_{\text {out }}}-1}{2 g_{n}^{S D} g_{n}^{R D}}}
\end{aligned}
$$

- Calculate $e_{n}^{\text {direct }}\left(\kappa_{n}\right)$ using (5). If $e_{n}^{\text {direct }}<e_{n}^{S D}\left(\kappa_{n}\right)+$ $e_{n}^{R D}\left(\kappa_{n}\right)+e_{n}^{S R}\left(\kappa_{n}\right)$

$$
\begin{aligned}
& e_{n}^{S D}\left(\kappa_{n}\right)=e_{n}^{\text {direct }}\left(\kappa_{n}\right) \\
& e_{n}^{R D}\left(\kappa_{n}\right)=e_{n}^{S R}\left(\kappa_{n}\right)=0
\end{aligned}
$$

\section{Amplify and Forward}

In this scheme, in the first subframe the source transmits to the relay and the destination also hears it. In the second subframe the relay amplifies and forwards the received signal. At the same time the source transmits again. The source and relay use space time coding as mentioned in [9],[20]. Frame model is similar to the DF model in Figure 1(b). AF is simpler than DF since there is no decoding at the relay. Besides, its performance can be better since there is no error propagation by decoding at the relay. In this work we aim to find the minimum energy power allocation that satisfies the rate and outage probability constraints. Let us define $\gamma_{n}^{S D}=$ $\frac{e_{n}^{S D} g_{n}^{S D} h^{S D}}{T_{f} N_{o} W_{s} \kappa_{n} / 2}, \gamma_{n}^{R D}=\frac{e_{n}^{R D} g_{n}^{R D} h^{R D}}{T_{f} N_{o} W_{s} \kappa_{n} / 2}, \quad \gamma_{n}^{S R}=\frac{e_{n}^{S R} g^{S R} h^{S R}}{T_{f} N_{o} W_{s} \kappa_{n} / 2}$ and $\gamma_{n}^{S R D}=\frac{e_{n}^{S R} g^{S D} h^{S D}}{T_{f} N_{o} W_{s} \kappa_{n} / 2}$. Assuming $\kappa_{n}$ subchannels are allocated, the mutual information at the receiver can be found as $[20]^{1}$,

$$
\begin{aligned}
I=\frac{1}{2} \log _{2}(1 & +\frac{1}{\omega^{2}} \gamma_{n}^{S D}+\gamma_{n}^{S R D} \\
& \left.+\frac{1}{\omega^{2}} \gamma_{n}^{S R D} \gamma_{n}^{S D}+\frac{\gamma_{n}^{S R} \gamma_{n}^{R D}}{1+\gamma_{n}^{S R}+\gamma_{n}^{R D}}\right)
\end{aligned}
$$

${ }^{1}$ In [20] it was assumed that $h^{R D}=1$, while deriving the mutual information and outage. In this work we assume that it is also exponentially distributed like $h^{S D}$ and $h^{S R}$. 
where $\omega^{2}=1+\frac{\gamma_{n}^{R D}}{\gamma_{n}^{S R}+1}$. The term $\frac{1}{\omega^{2}} \gamma_{n}^{S R D} \gamma_{n}^{S D}$ involves $\left(h^{S D}\right)^{2}$, which is small for the outage case, therefore it is ignored [20]. One more approximation can be made. Sourcerelay channel is usually much better than the relay destination channel. Therefore $\omega^{2}$ can be approximated as 1 . Outage probability at the receiver of destination $n$ can be written as,

$$
\begin{aligned}
\frac{P_{\text {out }}}{\kappa_{n}}= & \operatorname{Pr}\left\{\frac{\frac{e_{n}^{S R} g^{S R} h^{S R}}{T_{f} N_{o} W_{s} \kappa_{n} / 2} \frac{e_{n}^{R D} g_{n}^{R D} h^{R D} N_{o} W_{s} \kappa_{n} / 2}{T_{n}^{S R}}}{1+\frac{e_{n}^{S R} h^{S R} h^{S R}}{T_{f} N_{o} W_{s} \kappa_{n} / 2}+\frac{e_{n}^{R D} g_{n}^{R D} h^{R D}}{T_{f} N_{o} W_{s} \kappa_{n} / 2}}\right. \\
+ & \left.\frac{\left(e_{n}^{S R}+e_{n}^{S D}\right) g_{n}^{S D} h^{S D}}{T_{f} N_{o} W_{s} \kappa_{n} / 2}<2^{\frac{2 R_{n}}{T_{f} W_{s} \kappa_{n}}}-1\right\}
\end{aligned}
$$

Here we will neglect the "1" in the denominator, which is a common high-SNR assumption [4][11]. Then, defining $e_{n}^{0}\left(\kappa_{n}\right)=0.5 T_{f} \kappa_{n} N_{o} W_{s}\left(2^{\frac{2 R_{n}}{W_{s} T_{f} \kappa_{n}}}-1\right)$ as before, we get,

$$
\begin{aligned}
\frac{P_{\text {out }}}{\kappa_{n}}=\operatorname{Pr}\{( & \left.\frac{1}{e_{n}^{S R} g^{S R} h^{S R}}+\frac{1}{e_{n}^{R D} g_{n}^{R D} h^{R D}}\right)^{-1} \\
& \left.+\left(e_{n}^{S R}+e_{n}^{S D}\right) g_{n}^{S D} h^{S D}<e_{n}^{0}\left(\kappa_{n}\right)\right\}
\end{aligned}
$$

The first term involves the harmonic mean of two exponential random variables with means $e_{n}^{S R} g^{S R}$ and $e_{n}^{R D} g_{n}^{R D}$, respectively. In [21] it was proven that for the high SNR case the harmonic mean of two exponential random variables can be approximated by an exponential random variable with mean equal to the harmonic mean of the two means. This approximation is successful especially in outage calculations. Now, as before, outage event involves the summation of two exponential random variables being smaller than a constant. Using the result in (10), we obtain,

$$
\frac{e_{n}^{S R}+e_{n}^{S D}}{\frac{1}{e_{n}^{S R} g^{S R}}+\frac{1}{e_{n}^{R D} g_{n}^{R D}}}=\frac{\left(e_{n}^{0}\left(\kappa_{n}\right)\right)^{2} \kappa_{n}}{2 g_{n}^{S D} P_{\text {out }}}
$$

In order to find the minimum-energy allocation that satisfies the above equation, we formulate a constrained optimization problem of minimizing $e_{n}^{S R}+e_{n}^{R D}+e_{n}^{S D}$ subject to the satisfaction of equality (21). We obtain the energy values as a function of number of allocated subchannels (details are omitted for the sake of brevity)

$$
\begin{aligned}
& \text { If } g^{S R}<g_{n}^{R D} \text {, } \\
& e_{n}^{S R}\left(\kappa_{n}\right) \\
& =e_{n}^{0}\left(\kappa_{n}\right) \sqrt{\frac{0.5 \kappa_{n}}{g_{n}^{S D} P_{\text {out }}} \frac{\sqrt{g_{n}^{R D}}}{g^{S R}}\left(\frac{1}{\sqrt{g^{S R}}}+\frac{1}{\sqrt{g_{n}^{R D}}}\right)} \\
& e_{n}^{R D}\left(\kappa_{n}\right) \\
& =e_{n}^{0}\left(\kappa_{n}\right) \sqrt{\frac{0.5 \kappa_{n}}{g_{n}^{S D} P_{\text {out }}} \frac{1}{\sqrt{g_{n}^{R D}}}\left(\frac{1}{\sqrt{g^{S R}}}+\frac{1}{\sqrt{g_{n}^{R D}}}\right)}
\end{aligned}
$$

$$
\begin{aligned}
& e_{n}^{S D}\left(\kappa_{n}\right)= \\
& e_{n}^{0}\left(\kappa_{n}\right) \sqrt{\frac{0.5 \kappa_{n}}{g_{n}^{S D} P_{\text {out }}}\left(\frac{1}{\sqrt{g^{S R}}}+\frac{1}{\sqrt{g_{n}^{R D}}}\right)\left(\frac{1}{\sqrt[4]{g_{n}^{R D}}}-\frac{\sqrt[4]{g_{n}^{R D}}}{\sqrt{g^{S R}}}\right)} \\
& \quad \text { If } g^{S R}<g_{n}^{R D} \\
& e_{n}^{S R}\left(\kappa_{n}\right) \\
& =e_{n}^{0}\left(\kappa_{n}\right) \sqrt{\frac{0.5 \kappa_{n}}{g_{n}^{S D} P_{\text {out }}} \frac{1}{\sqrt{g^{S R}}}\left(\frac{1}{\sqrt{g^{S R}}}+\frac{1}{\sqrt{g_{n}^{R D}}}\right)} \\
& \quad=e_{n}^{0}\left(\kappa_{n}\right) \sqrt{\frac{0.5 \kappa_{n}}{g_{n}^{S D} P_{\text {out }}} \frac{\sqrt{g^{S R}}}{g_{n}^{R D}}\left(\frac{1}{\sqrt{g^{S R}}}+\frac{1}{\sqrt{g_{n}^{R D}}}\right)} \\
& e_{n}^{S D}\left(\kappa_{n}\right)=0
\end{aligned}
$$

After these calculations we again calculate $e_{n}^{\text {direct }}\left(\kappa_{n}\right)$ using (5). If $e_{n}^{\text {direct }}<e_{n}^{S D}\left(\kappa_{n}\right)+e_{n}^{R D}\left(\kappa_{n}\right)+e_{n}^{S R}\left(\kappa_{n}\right)$

$$
\begin{aligned}
& e_{n}^{S D}\left(\kappa_{n}\right)=e_{n}^{\text {direct }}\left(\kappa_{n}\right) \\
& e_{n}^{R D}\left(\kappa_{n}\right)=e_{n}^{S R}\left(\kappa_{n}\right)=0
\end{aligned}
$$

D. Decode and Forward with Time Optimization (DF-TO)

Time optimization can be used together with cooperative transmission as in Figure 1(c). In the first subframe the source transmits to the relay but the destination doesn't decode this signal, therefore the modulation/coding pair - hence, the duration- can be different than $T_{f} / 2$. In the second subframe the source and relay transmit simultaneously using again a space time coding scheme. We call this scheme as Decodeand-Forward with Time Optimization (DF-TO). Now let us define, $e_{n}^{01}\left(\kappa_{n}, \alpha_{n}\right)=\alpha_{n} T_{f} \kappa_{n} N_{o} W_{s}\left(2^{\frac{R_{n}}{W_{s} T_{f} \kappa_{n} \alpha_{n}}}-1\right)$ and $e_{n}^{02}\left(\kappa_{n}, \alpha_{n}\right)=\left(1-\alpha_{n}\right) T_{f} \kappa_{n} N_{o} W_{s}\left(2^{\frac{R_{n}}{W_{s} T_{f} \kappa_{n}\left(1-\alpha_{n}\right)}}-1\right)$. We redefine the subchannel outage probability as follows [3],

$$
\begin{gathered}
\frac{P_{\text {out }}}{\kappa_{n}}=\operatorname{Pr}\left\{e_{n}^{S R} g^{S R} h^{S R}<e_{n}^{01}\left(\kappa_{n}, \alpha_{n}\right)\right\} \\
+\operatorname{Pr}\left\{e_{n}^{S R} g^{S R} h^{S R} \geq e_{n}^{01}\left(\kappa_{n}, \alpha_{n}\right)\right\} \\
\times \operatorname{Pr}\left\{e_{n}^{S D} g_{n}^{S D} h^{S D}+e_{n}^{R D} g_{n}^{R D} h^{R D}<e_{n}^{02}\left(\kappa_{n}, \alpha_{n}\right)\right\}
\end{gathered}
$$

As before, we target $\operatorname{Pr}\left\{e_{n}^{S R} g^{S R} h^{S R}<e_{n}^{01}\left(\kappa_{n}, \alpha_{n}\right)\right\}=$ $\frac{P_{\text {out }}}{2 \kappa_{n}}$. We directly find $e_{n}^{S R}$ as,

$$
e_{n}^{S R}\left(\kappa_{n}, \alpha_{n}\right)=\frac{e_{n}^{01}\left(\kappa_{n}, \alpha_{n}\right)}{-\ln \left(1-\frac{P_{\text {out }}}{2 \kappa_{n}}\right) g^{S R}}
$$

Other term involves the summation of two exponentially distributed random variables being smaller than a constant.

$$
\frac{1}{\frac{2 \kappa_{n}}{P_{\text {out }}}-1}=\frac{\frac{1-e^{-\frac{e_{n}^{02}\left(\kappa_{n}, \alpha_{n}\right)}{e_{n}^{S D} g_{n}^{S D}}}}{e_{n}^{R D} g_{n}^{R D}}-\frac{1-e^{-\frac{e_{n}^{02}\left(\kappa_{n}, \alpha_{n}\right)}{e_{n}^{R D} g_{n}^{R D}}}}{\left(e_{n}^{S R}+e_{n}^{S D}\right) g_{n}^{S D}}}{\frac{1}{e_{n}^{R D} g_{n}^{R D}}-\frac{1}{\left(e_{n}^{S R}+e_{n}^{S D}\right) g_{n}^{S D}}}
$$


Again, we make the approximation $e^{-x} \simeq 1-x+x^{2}$ and write the following

$$
\frac{1}{\frac{2 \kappa_{n}}{P_{\text {out }}}-1}=\frac{\left(e_{n}^{02}\left(\kappa_{n}, \alpha_{n}\right)\right)^{2}}{2 e_{n}^{S D} g_{n}^{S D} e_{n}^{R D} g_{n}^{R D}}
$$

If we use the approximation in (10) then

$$
e_{n}^{S D}\left(\kappa_{n}, \alpha_{n}\right)=e_{n}^{R D}\left(\kappa_{n}, \alpha_{n}\right)=e_{n}^{02}\left(\kappa_{n}, \alpha_{n}\right) \sqrt{\frac{\frac{2 \kappa_{n}}{P_{\text {out }}}-1}{2 g_{n}^{S D} g_{n}^{R D}}}
$$

Let $\alpha_{n}^{*}$ be the value that minimizes $e_{n}^{\text {relay }}\left(\kappa_{n}, \alpha_{n}\right)=$ $e_{n}^{S D}\left(\kappa_{n}, \alpha_{n}\right)+e_{n}^{S D}\left(\kappa_{n}, \alpha_{n}\right)+e_{n}^{S D}\left(\kappa_{n}, \alpha_{n}\right)$. Power expenditure by direct transmission can be found using (5). If $e_{n}^{\text {direct }}\left(\kappa_{n}\right)$ is smaller than $e_{n}^{\text {relay }}\left(\kappa_{n}, \alpha_{n}^{*}\right)$ then direct transmission is chosen.

\section{E. Resource Allocation Algorithm}

While describing the above transmission schemes, we derived the energy (and subframe time) allocation given the number of subchannels allocated to a user. How do we determine the number of subchannels allocated to each user? In this section we present the subchannel allocation algorithm for the DF-TO scheme. Other schemes are similar,

1) Set $\kappa_{n}=1, \forall n$

2) For each user calculate $e_{n}\left(\kappa_{n}, \alpha_{n}^{*}\right)$ and $e_{n}\left(\kappa_{n}+1, \alpha_{n}^{*}\right)$ $\left(e_{n}\left(\kappa_{n}, \alpha_{n}^{*}\right)=\min \left\{e_{n}^{\text {relay }}\left(\kappa_{n}, \alpha_{n}^{*}\right), e_{n}^{\text {direct }}\left(\kappa_{n}\right)\right\}\right)$

3) Determine $n^{*}=\arg \max _{n}\left\{e_{n}\left(\kappa_{n}, \alpha_{n}^{*}\right)-e_{n}\left(\kappa_{n}+\right.\right.$ $\left.\left.1, \alpha_{n}^{*}\right)\right\}$ and $\Delta e^{*}=\max _{n}\left\{e_{n}\left(\kappa_{n}, \alpha_{n}^{*}\right)-e_{n}\left(\kappa_{n}+1, \alpha_{n}^{*}\right)\right\}$

4) If $\Delta e^{*}>0$ Set $\kappa_{n^{*}}=\kappa_{n^{*}}+1$ else finish

5) If $\sum_{n} \kappa_{n}=K$ finish else continue from Step 2

The proposed greedy algorithm allocates at each round a subchannel to the user that most needs it. Step 1 allocates each user a subchannel. Step 2 calculates the energy requirement for the current number of subchannels and for the case of one more added subchannel for each user. Step 3 calculates the decrease in energy expenditure by adding one more subchannel to each user. Then it finds the user that can most improve its energy expenditure by adding a subchannel. Step 4 allocates a subchannel to the maximizing user. This continues until all subchannels are allocated. Depending on the characteristics of the energy function it is also possible that allocating more than a certain number of subchannels causes increase in energy expenditure. This is also the case in our problem and it is related to the definition of outage. An outage happens for a destination node if one of the subchannels experience outage. Increasing the number of subchannels first decreases the energy expenditure but then increases the chance of outage (Figure 2), thus requiring more energy to compensate it (especially if rate requirement is low). The algorithm finishes if either all subchannels are allocated or adding a subchannel results in increased energy expenditure for all nodes.

Step 2 also involves finding the optimal $\alpha_{n}$ for a given number of allocated subchannels for all users (For NCRTO and DF-TO). Simulation results show that the energy $e_{n}\left(\kappa_{n}, \alpha_{n}\right)$ is a concave function of $\alpha_{n}=t_{n} \times \frac{T_{s}}{T_{f}}$ where $t_{n} \in\{1,2, \ldots, K-1\}$. The search algorithm starts from

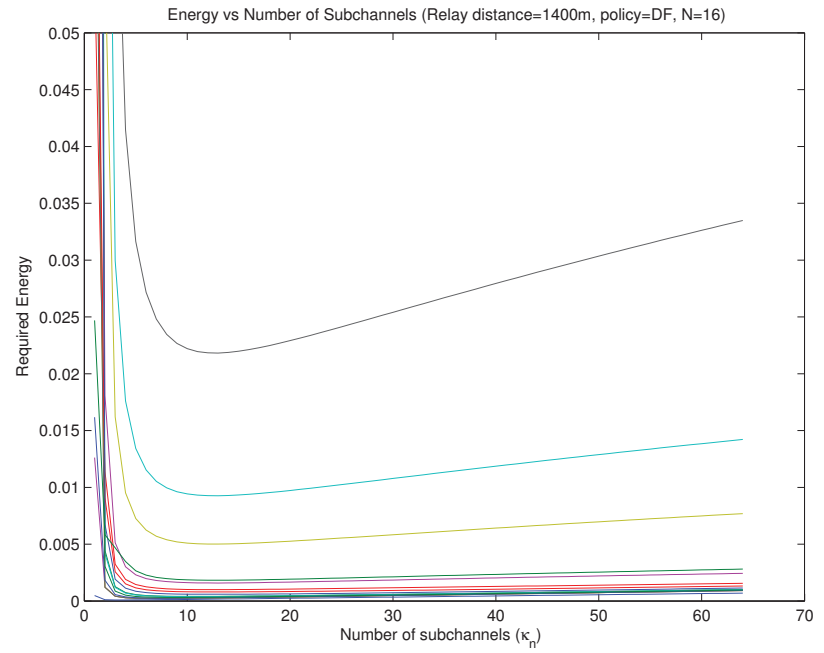

Fig. 2. Variation of required energy expenditure for all users with the number of subchannels. The characteristics show that adding one more subchannel brings diminishing returns as the number of subchannels increase. In fact energy expenditure increases after allocating more than a certain number of subchannels to a node. The characteristics are similar for all transmission schemes.

$t_{n}=\operatorname{round}\left(\frac{T_{f}}{T_{s} 2}\right)$. Then the algorithm increases $t_{n}$ by one. If the energy expenditure decreases, then $t_{n}$ is increased by one until the minimizing $t_{n}$ is found. If the energy expenditure increases then $t_{n}$ is decreases by one until the minimizing $t_{n}$ is found.

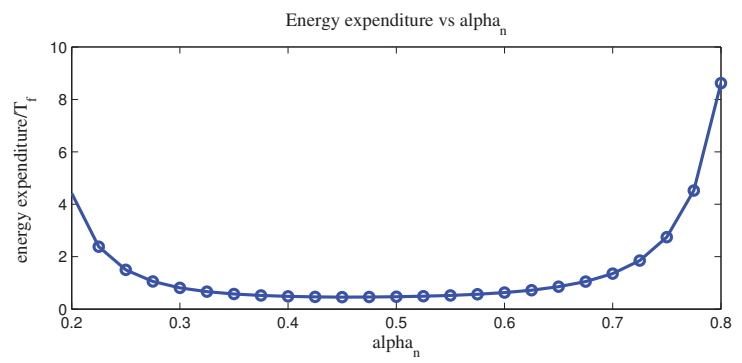

Fig. 3. Energy expenditure is a convex function of $\alpha_{n}$. Giving too much subframe for either source or relay increases the energy requirement of the other one. Optimal $\alpha_{n}$ is somewhere in between. This convexity simplifies search for energy-optimal $\alpha_{n}^{*}$. Relay distance is $1400 \mathrm{~m}$, number of nodes is 16. Total rate requirement is $10 \mathrm{Mbps}$.

1) Optimality: The resource allocation algorithm greedily allocates the subchannels to the user that it provides most energy decrease. The paper [22] proves that such greedy allocations are optimal for a specific class of problems. If adding more resources to a node brings diminishing returns (i.e. concavity), then allocating a resource to the user that brings maximum increase of utility is the optimum scheme. In our problem we can regard negative energy as the utility. Diminishing return characteristics in Figure 2 show that the above algorithm is suitable. On the other hand, various ap- 
proximations that we made to ease the analysis results in loss of optimality.

\section{Numerical EVAluation}

In this section we numerically evaluate the four transmission policies. We consider a tandem cell of 2000 meters long, where the base station is located at the origin and the users are located uniformly in the region 100-2000 meters. As for the channel model we adopt the IEEE $802.16 \mathrm{j}$ model, where the path loss in the source relay (BS-RS) link is $31.5+35 \times \log _{10}(d) \mathrm{dB}$ and the path loss in the source-destination and relay-destination link is $36.5+23.5 \times \log _{10}(d) \mathrm{dB}$. Here $d$ (in meters)is the distance of the considered link. Log normal shadowing (in $\mathrm{dB}$ ) has standard deviation of $3.4 \mathrm{~dB}$ in the source relay and $8 \mathrm{~dB}$ in the source-destination and relay destination channel. Rayleigh fading is assumed to be independent across all the links and subchannels. Total bandwidth is $5 \mathrm{MHz}$ and it is divided into 64 subchannels.

Figure 4 presents the total energy expenditure vs. distance of relay from the base station. We obtained these results for a total rate requirement of $10 \mathrm{Mbps}$ divided equally into 16 users. We changed the source-relay distances from 1000 to $1800 \mathrm{~m}$ in $100 \mathrm{~m}$ steps. For each distance we generated 10000 different sets of path losses, shadow fadings and Rayleigh fadings; ran the algorithms and took the averages of total energy expenditures. We further divided these values by the frame duration $T_{s}$ to obtain the average power expenditure. The most interesting result in Figure 4 is that cooperative transmission results in significant savings from energy. Cooperative relaying results in less than half (almost one third) of the energy expenditure of noncooperative relaying. Second interesting result is that the further performance improvement by optimization of the subframe time may not be worth it because it is less significant and requires much more computation time. In fact AF without time optimization results in approximately 30 percent less energy expenditure than DF and 15-20 percent less energy expenditure than DF-TO. Lastly, we observe that best performance is observed when the source-relay distance is between $1400-1500 \mathrm{~m}$.

We also need to check if we achieve the target outage probability. Target outage probability is chosen to be 0.01 in all simulations. Table I shows that for the non cooperative case this target is achieved. For the cooperative cases the outage performance is better than 0.01 . This is because of the approximation $e^{-x} \simeq 1-x+x^{2}$ that was made in the analysis. Less energy expenditure can be achieved by using the exact formula instead of approximations, however the approximation results in a very simple scheme with acceptable performance. In the AF scheme we make more approximations which results in more deviations from 0.01 .

The reason of close performance of CR and CR-TO implies that time optimization is not as effective as cooperative diversity. Figure 3 shows for a typical case that the performance $0.3<\alpha_{n}<0.6$ does not provide significant performance variation. Figure 5 shows the cumulative distribution of $\alpha$ for the above simulation parameters. This figure shows that

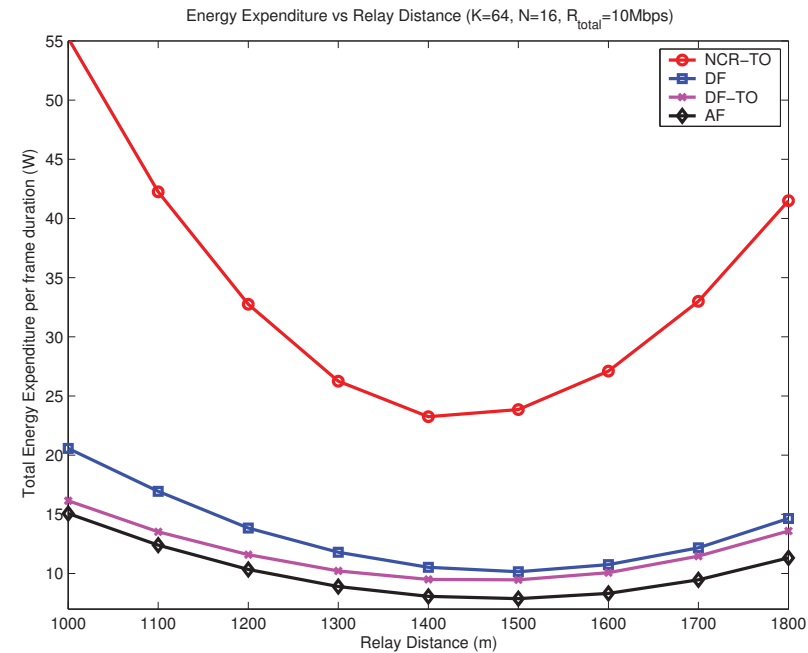

Fig. 4. Energy expenditure performances for all four policies as a function of relay distance from base station. Number of users is 16 and total rate requirement is $10 \mathrm{Mbps}$. $\mathrm{AF}$ and $\mathrm{DF}$ protocols are simple since they do not need time optimization. Besides their performance is much better than non cooperative scheme. Here, AF algorithm results in the best performance probably since it does not propagate errors.

\begin{tabular}{|c|c|c|c|c|c|}
\hline & $1000 \mathrm{~m}$ & $1200 \mathrm{~m}$ & $1400 \mathrm{~m}$ & $1600 \mathrm{~m}$ & $1800 \mathrm{~m}$ \\
\hline \hline NCR-TO & 0.0103 & 0.0102 & 0.00954 & 0.0103 & 0.00998 \\
\hline CR & 0.00917 & 0.0874 & 0.00938 & 0.00921 & 0.00941 \\
\hline CR-TO & 0.00900 & 0.00849 & 0.00875 & 0.00877 & 0.00858 \\
\hline AF & 0.00844 & 0.00804 & 0.00816 & 0.00810 & 0.00825 \\
\hline
\end{tabular}

TABLE I

Outage Probabilities vs. Distance for ALl POLICIES.

$0.3<\alpha_{n}<0.6$ has an 80 percent occurrence, which means that for most of the cases optimizing $\alpha$ results in insignificant performance difference.

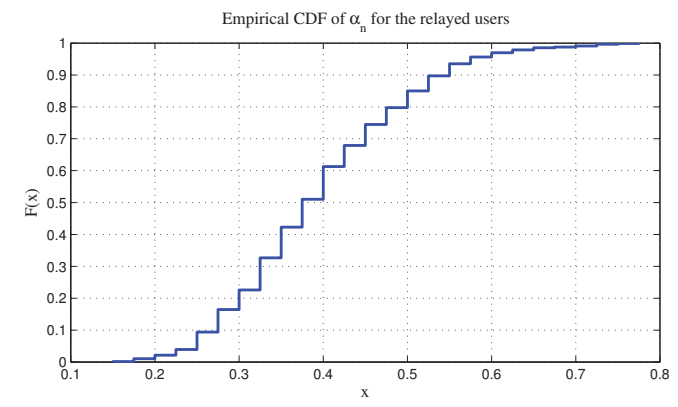

Fig. 5. Cumulative distribution of alpha for the relayed users. Source-Relay distance $1400 \mathrm{~m} . \mathrm{N}=16$

Figure 6 shows the cumulative distribution of the total energy expenditure per frame for all four policies. We see that the AF policy is clearly the best one. Figure 7 shows the total energy expenditure per frame as a function of number of users. Total rate requirement is $10 \mathrm{Mbps}$ and is divided equally among the users. Energy expenditure decreases 
because rate requirement per user decreases, although the total rate requirement keeps the same.

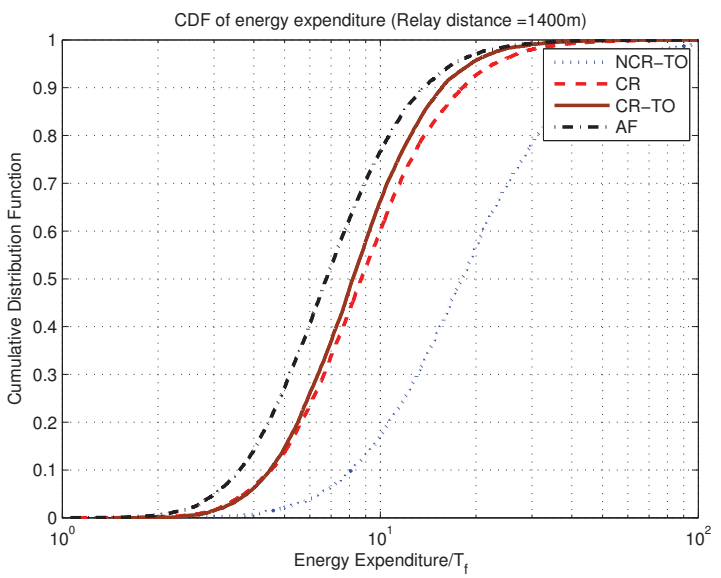

Fig. 6. Cumulative distribution of total energy expenditure (normalized by frame duration). Source-Relay distance 1400m. N=16

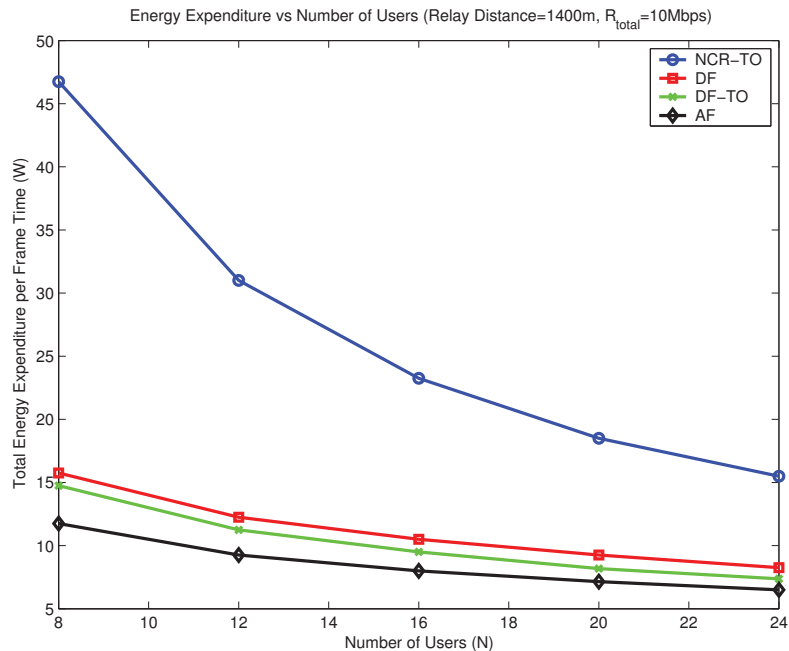

Fig. 7. Total energy expenditure per frame vs number of users. Total rate requirement is $10 \mathrm{Mbps}$. Source-Relay distance $1400 \mathrm{~m}$.

\section{CONCLUSION}

In this paper we proposed an energy-efficient subchannel, power and subframe allocation scheme for a multiuser downlink OFDMA system that utilizes a relay node. The source makes the resource allocation only based on the pathloss and slow fading, without knowing the fast fading. In this setting, we showed that using cooperative diversity both achieves the target outage probability and requires less than half, almost one-third of energy required by non-cooperative relaying. This performance improvement can be achieved without adjusting the subframe durations, which significantly decreases the complexity. Future work will consider Incremental Relaying and other more complex policies [3] in addition to Decode and Forward and Amplify and Forward. Proportional Fairness will be considered as an objective instead of energy efficiency.

\section{ACKNOWLEDGMENT}

The work in this paper was supported by TUBITAK under 1001 grant 108E208.

\section{REFERENCES}

[1] Masato Okuda, Chenxi Zhu, Dorin Viorel,'Multihop Relay Extention for WiMax Networks-Overview and Benefits of 802.16j IEEE Standard", Fujitsu scientific and technical journal, vol. 44, no. 3, pp. 292-302, 2008

[2] Pabst, R.; Walke, B.H.; Schultz, D.C.; Herhold, P.; Yanikomeroglu, H.; Mukherjee, S.; Viswanathan, H.; Lott, M.; Zirwas, W.; Dohler, M.; Aghvami, H.; Falconer, D.D.; Fettweis, G.P., "Relay-based deployment concepts for wireless and mobile broadband radio," Communications Magazine, IEEE , vol.42, no.9, pp. 80-89, Sept. 2004

[3] J. N. Laneman , D. N. C. Tse and G. W. Wornell Cooperative diversity in wireless networks: Efficient protocols and outage behavior, IEEE Trans. Inf. Theory, vol. 50, pp. 30-62, Dec. 2004.

[4] Yong Li; Wenbo Wang; Jia Kong; Wei Hong; Xing Zhang; Mugen Peng, "Power Allocation and Subchannel Pairing in OFDM-Based Relaying Networks", IEEE International Conference on Communications pp.26022606, 19-23 May 2008

[5] Lei You; Mei Song; Junde Song; Qingyu Miao; Yong Zhang, ”Adaptive Resource Allocation in OFDMA Relay-Aided Cooperative Cellular Networks," Vehicular Technology Conference, pp.1925-1929, 11-14 May 2008

[6] O. Amin and M. Uysal, Adaptive Power Loading for OFDM Cooperative Networks, 11th Canadian Workshop on Information Theory, Ottawa, Ontario, Canada, May 2009

[7] Truman Chiu-Yam Ng, Wei Yu, Jianzhong Zhang, Anthony Reid "Joint Optimization of Relay Strategies and Resource Allocations in Cooperative Cellular Networks"IEEE Journal on Selected areas in Communications, February, 2007. pp. 328-339.

[8] Can, B; Yanikomeroglu, H.; Onat, F.A.; De Carvalho, E.; Yomo, H., "Efficient Cooperative Diversity Schemes and Radio Resource Allocation for IEEE 802.16j," IEEE Wireless Communications and Networking Conference, pp.36-41, March 31 -April 32008

[9] B. Can, H. Yomo, and E. d. Carvalho, "Link Adaptation and Selection Method for OFDM Based Wireless Relay Networks," Journal of Communication Networks, vol. 9, no. 2, pp. 118-127, June 2007

[10] Bo Gui; Lin Dai; Cimini, L.J., "Selective Relaying in Cooperative OFDM Systems: Two-Hop Random Network," Wireless Communications and Networking Conference, 2008. WCNC 2008. IEEE, vol., no., pp.9961001, March 31 2008-April 32008

[11] Siriwongpairat, W.; Sadek, A.; Liu, K.J.R., "Cooperative communications protocol for multiuser OFDM networks," Wireless Communications, IEEE Transactions on , vol.7, no.7, pp.2430-2435, July 2008

[12] K. Bakanoglu, D. Gunduz, and E. Erkip, Dynamic resource allocation for the broadband relay channel, in Proc. Asilomar Conf. on Signals, Systems and Computers, Monterey, CA, Nov. 2007.

[13] Kibeom Seong, Mehdi mohseni, John M. Cioffi "Optimal Resource Allocation for OFDMA Downlink Systems" IEEE ISIT, July 9-14th, 2006.

[14] Inhyoung Kim, In-Soon Park, Lee, Y.H. "Use of linear programming for dynamic subchannel and bit allocation in multiuser OFDM" IEEE Veh. Technol. Vol. 55 pp. 1195-120

[15] T. Girici "Joint power, subchannel and subframe allocation in Multihop relay networks", International Journal of Communication Systems, vol. 22(7), pp. 835-855, 2009.

[16] C. Muller, A. Klein, F. Wegner, M. Kuipers, and B. Raaf, "Dynamic Subchannel, Bit and Power Allocation in OFDMA-Based Relay Networks," in Proceedings of 12th International OFDM Workshop, 2007.

[17] Kaneko, M.; Popovski, P.; Hayashi, K., 'Throughput-Guaranteed Resource-Allocation Algorithms for Relay-Aided Cellular OFDMA System," Vehicular Technology, IEEE Transactions on , vol.58, no.4, pp.1951-1964, May 2009 
[18] Z. Han; T. Himsoon; W.P. Siriwongpairat; K.J.R. Liu, ’Energy-efficient cooperative transmission over multiuser OFDM networks: who helps whom and how to cooperate," IEEE Wireless Communications and Networking Conference, vol.2, pp. 1030-1035 , 13-17 March 2005

[19] B. Gui,L. J. Cimini Jr. "Bit Loading Algorithms for Cooperative OFDM Systems", EURASIP Journal onWireless Communications and Networking, Volume 2008, p.p 1-9.

[20] R. U. Nabar, H. Blcskei, and F. W. Kneubuhler Fading relay channels: Performance limits and space-time signal design IEEE J. Sel. Areas Commun., vol. 22, no. 6, pp. 1099-1109, Aug. 2004.

[21] Seddik, K.G.; Sadek, A.K.; Weifeng Su; Liu, K.J.R., "Outage Analysis and Optimal Power Allocation for Multinode Relay Networks," Signal Processing Letters, IEEE, vol.14, no.6, pp.377-380, June 2007

[22] W. Shih, "A New Application of Incremental Analysis in Resource Allocations", Journal of the Operational Research Society (1974) 25, 587597 